\title{
New path towards a better rice architecture
}

\author{
Cell Research (2017) 27:1189-1190. doi:10.1038/cr.2017.115; published online 12 September 2017
}

\begin{abstract}
New plant type (NPT) or ideal plant architecture (IPA) is an attractive way of increasing yield potential by promoting high resource use efficiency combined with better lodging resistance. In a recent paper in Cell Research, Wang et al. describe how a QTL they identified could bring about the desired NPT architecture by elucidating the role of its encoded gene in controlling the stability of IPA1/OsSPL14, a previously reported NPT protein, in the context of ubiquitination.
\end{abstract}

Decades after the Green Revolution, plant breeders are still faced with the challenge to increase the yield potential of rice for the sake of sustaining a booming global population [1]. One of the popular approaches used by breeders to attain higher yield is to breed plants with ideal plant architecture, leading to the New Plant Type (NPT) concept. Unlike the semi-dwarf plants of the Green Revolution [2, 3], NPT plants have less tillers but more grains per panicle coupled with sturdier stems, giving rise to higher yield potential and better lodging resistance.

In a recent paper in Cell Research, Wang et al. [4] report a gene involved in the NPT architecture. The gene was identified through QTL analysis and positional cloning using japonica-indica crosses and was named as rice $O T U B 1$ (OSOTUB1) because of its homology to human OTUB1 (ovarian tumor domain-containing ubiquitin aldehydebinding protein 1). Plants showing the NPT phenotype had five unique DNA polymorphisms in OSOTUB1 relative to conventional plants, and such polymorphisms did not alter the amino acid se- quence. The involvement of OSOTUB1 in the NPT architecture was confirmed by the following observations. First, a near isogenic line (NIL) carrying an allele with downregulated OSOTUB1 expression showed the typical NPT phenotypes such as enhanced size of the shoot apical meristem (SAM), increased grain number per panicle due to increased primary and secondary rachis number, increased culm diameter and 1000 -grain weight, and reduced tiller number per plant. Second, similar phenotypes were observed in plants with disrupted OSOTUB1 function by CRISPR/Cas9. Third, Huang et al. [5] also recently reported the null allele mutant (wtgl) of OsOTUB1 and the same grain phenotypes as OSOTUBI loss-of-function mutants were observed, further confirming that OSOTUB1 negatively regulates these traits. In contrast, OsOTUB 1 overexpression induced aberrant phenotypes such as dwarfism, malformed panicle with decreased grain number, necrotic leaves, and decreased tiller number per plant, which are not completely opposite to the phenotypes of the loss-of-function mutants, and thus, may indicate the side-effect of OsOTUB1 overproduction.

Previous studies revealed that the human OTUB1 is a Lysine48 (K48)specific deubiquitinating enzyme [6], whereas this protein was also reported to directly interact with the ubiquitin (Ub)-conjugating enzyme (E2), UBC13, thereby inhibiting ubiquitin transfer [7]. In contrast to human OTUB1, OsOTUB1 has cleavage activity for both $\mathrm{K} 48$ - and K63-linked Ub chains; but like human OTUB1, OsOTUB1 also physically interacts with rice UBC13 (OsUBC13).
Overexpression of $O S U B C 13$ also induced the NPT phenotypes, whereas its knockdown plants showed similar phenotypes with OSOTUB1 overexpressors. They further searched for proteins interacting with OsOTUB1, and identified SQUAMOSA promoter-binding protein-like (SPL) transcription factor 14 (OsSPL14) or IPA1, which is known to control plant architecture associated with reduced tiller number, thickened culm and enhanced grain number $[8,9]$. They revealed that the NPT architecture controlled by $O S O T U B 1$ depends on IPA1/OSSPL14 function, and that the NPT architecture is governed by the antagonistic relationship between the two genes as OsOTUB1 promotes the degradation of IPA1/OsSPL14. One of the most interesting findings in this study is that OsOTUB1-mediated deubiquitination of K63-linked Ub chain is required for the degradation of IPA1/ OsSPL14, whereas the deubiquitination of K48-linked Ub-IPA1/OsSPL14 makes it resistant to proteasome-dependent degradation just like the case of human OTUB1. This is the reason why they called the cleavage of K63-linked poly$\mathrm{Ub}$ as a non-canonical type of regulation.

In relation to the Ub-mediated degradation of IPA1/OsSPL14, another IPA1/ OsSPL14-interacting protein, IPI1, a RING-finger E3 ligase was reported [10]. Just like OsOTUB1, overexpression and knockout of IPII causes decrease and increase in grain number per panicle, respectively, suggesting a possible similarity in their function in the Ub-mediated degradation of IPA1/OsSPL14 (Figure 1). Interestingly, IPI1 was found to have a tissue-specific dual nature; that is, it promotes proteasome-dependent degra- 


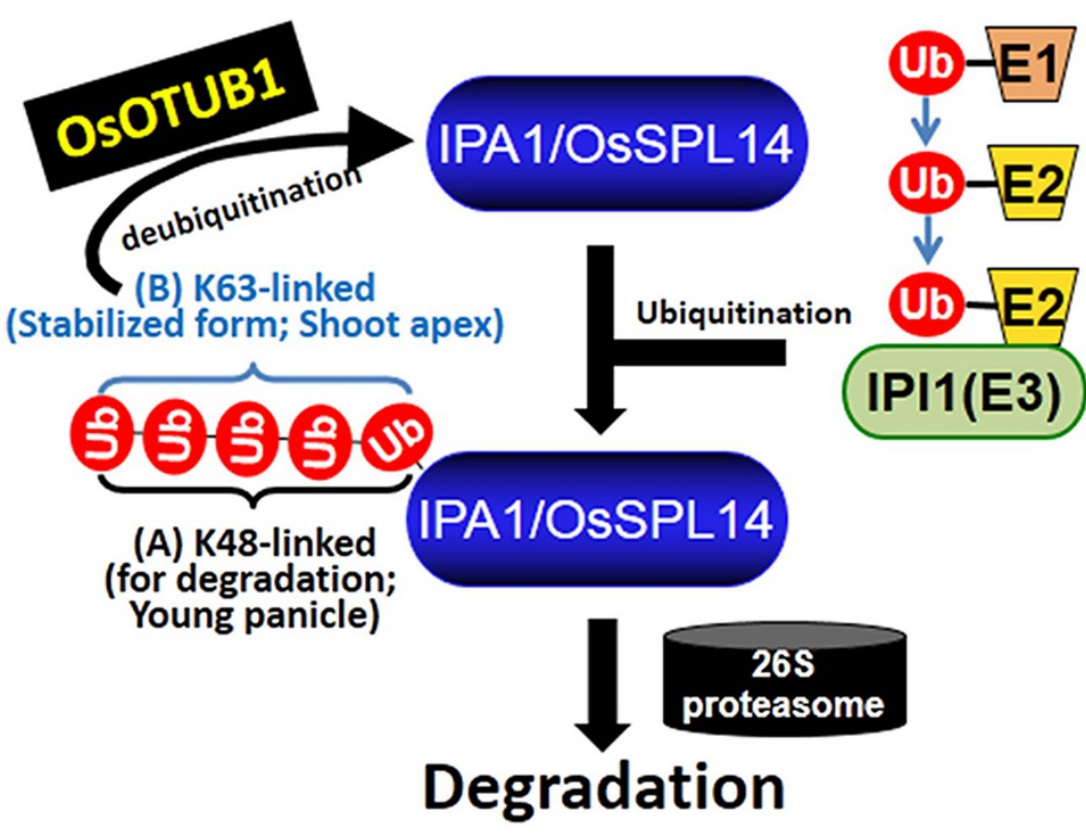

Figure 1 Model of IPA1/OsSPL14 degradation mediated by OsOTUB1 and IPI1 (E3). IPA1/OsSPL14 is a key factor regulating plant architecture in rice. Its ubiquitination is promoted by IPI1, an E3 ligase that can produce K48- or K63-linked Ub-IPA1/OsSPL14. The K48-linked type (A) mainly occurs in the young panicle and is degraded by the 26S-proteasome. In contrast, K63-linked type (B) mainly produced in the shoot apex resists proteasome-dependent degradation. OsOTUB1 has a deubiquitinating activity for the K63-linked poly-Ub, which promotes the production of K48-linked UbIPA1/OsSPL14 and consequently, leads to the degradation of IPA1/OsSPL14. In the case of loss of function of OsOTUB1 or IPI1, IPA1/OsSPL14 protein accumulates, leading to NPT plants.

dation of IPA1/OsSPL14 in young panicles and at the same time, promotes its stability in shoot apices. Consequently, IPII knockout plants showed increased tiller number compared to the IPA1/OsSPL14 overexpressors and OSOTUB1 mutants, which show decreased tiller number characteristic of NPT plants. About this dual nature of IPI1, Wang et $a l$. [10] explained that IPI1 ubiquitinates IPA1/OsSPL14 in two different manners; that is, K48-linked ubiquitination in panicles and K63-linked ubiquitination in shoot apices, which further supports the discussion above that IPA1/OsSPL14 with $\mathrm{K} 63$-linked $\mathrm{Ub}$ resists proteasomedependent degradation.

Every existing rice architectural concept has some drawbacks, and every attempt at increasing yield is subject to a trade-off. Indeed, most genes inducing large panicle, such as IPA1/OSSPL14 [4] and SCM3/OsTB1 [11], can increase the grain number per panicle, but at the same time, decrease the tiller number per plant. In the same manner, the OSOTUBI discussed by Wang et al. also appears to follow the same trade-off trend, but with good results in terms of high grain yield and lodging resistance. However, since breeders are under constant pressure to innovate, it is tempting to identify other genetic mechanisms that can break or attenuate such trade-off relationship in the future. To explore this possibility, the IPII discussed here could be a good candidate as it does not appear to sacrifice tiller number for increased grains per panicle. Furthermore, the paper about OsOTUB 1 by Wang et al. [4], alongside with the paper about IPII [10], strongly suggests that the mode of ubiquitination of IPA1/OsSPL14 (K48- or K63-linked) regulates its stability in an organ-specific manner and consequently controls the NPT or ideal rice architecture, although the detailed molecular mechanism for this has not yet been fully clarified. Further studies should therefore be done not only in terms of basic science dealing with the Ub-mediated proteasome pathway but also molecular breeding for us to find better ways of increasing rice yield.

Reynante L Ordonio ${ }^{1}$, Makoto Matsuoka ${ }^{2}$

${ }^{1}$ Plant Breeding and Biotechnology Division, Philippine Rice Research Institute, Maligaya, Science City of Munoz 3119, The Philippines; ${ }^{2}$ Bioscience and Biotechnology Center, Nagoya University, Chikusa, Nagoya 4648601 Correspondence: Makoto Matsuoka

Email: makoto@nuagr1.agr.nagoya-u.ac.jp

\section{References}

1 Hirano K, Ordonio RL, Matsuoka M. Proc Jpn Acad Ser B Phys Biol Sci 2017; 93:220233.

2 Sasaki A, Ashikari M, Ueguchi-Tanaka M, et al. Nature 2002; 416:701-702.

3 Spielmeyer W, Ellis MH, Chandler PM. Proc Natl Acad Sci USA 2002; 99:90439048.

4 Wang S, Wu K, Qian Q, et al. Cell Res 2017; 27:1142-1156.

5 Huang K, Wang D, Duan P, et al. Plant $J$ 2017; 91:849-860.

6 Sun X-X, Challagundla KB, Dai M-S. EMBO J 2012; 31:576-92.

7 Wiener R, Zhang X, Wang T, et al. Nature 2012; 483:618-622.

8 Jiao Y, Wang Y, Xue D, et al. Nat Genet 2010; 42:541-544.

9 Miura K, Ikeda M, Matsubara A, et al. Nat Genet 2010; 42:545-549.

10 Wang J, Yu H, Xiong G, et al. Plant Cell 2017; 29: 697-707.

11 Yano K, Ookawa T, Aya K, et al. Mol Plant 2015; 8:303-314. 